\title{
Evolution of Lobectomy for Lung Cancer: From Open to Robotic Surgery
}

\author{
Christiana Bithas ${ }^{1}$ and Amer Harky ${ }^{2 *}$ \\ ${ }^{1}$ Liverpool School of Medicine, UK \\ ${ }^{2}$ Department of Cardiothoracic Surgery, Liverpool Heart and Chest Hospital, UK
}

*Corresponding author: Amer Harky, Department of Cardiothoracic Surgery, Liverpool Heart and Chest Hospital Liverpool, UK

\begin{tabular}{|c|}
\hline ARTICLE INFO \\
\hline Received: 些 June 01, 2019 \\
\hline Published: 慧 June 21, 2019 \\
\hline Citation: Christiana Bithas, Amer Harky. \\
\hline $\begin{array}{l}\text { Evolution of Lobectomy for Lung Cancer: } \\
\text { From Open to Robotic Surgery. Biomed }\end{array}$ \\
\hline $\begin{array}{l}\text { J Sci \& Tech Res 19(1)-2019. BJSTR. } \\
\text { MS.ID.003244. }\end{array}$ \\
\hline
\end{tabular}

Keywords: Thoracic; Lobectomy; Lung Cancer; Open Surgery; Robotic Surgery

\begin{abstract}
The surgical approach to lung resection has continued to evolve over time, gaining a momentum of success with each surgical advance. A rapidly developing area is the singleport (uniport) video-assisted thoracoscopic surgery (VATS). The single-port approach has been in practice for wedge resections over a decade. In the last 10 years, single-port VATS has extended its application to major lung resections, including lobectomies for the resection of proven or suspected lung tumours. The use of a small surgical incision has proven to have benefits ranging from reduced post-operative pain to even being able to undergo these procedures without general anaesthesia. This has in turn led to the field of robotic thoracic surgery to gain a surge in popularity, which benefits also from the advantages of performing complex operations with the creation of a small incisions. With the shared goal of attaining a limited post-operative hospital stay, lower health care costs, reduced post-operative complications and increasing productivity; we aim to review the reported literature of current use of each surgical technique to perform lobectomies and the development in their ability to accomplish these.
\end{abstract}

\section{Introduction}

The development of minimally invasive techniques for lung resection have followed a thrilling and complicated course. Although thoracoscopy is at present viewed as a modern advance in lung cancer surgeries, the groundwork was laid by the Swedish physician Jacobeus in the late $19^{\text {th }}$ century, in the same period that the first elective open lobectomies were attempted [1]. In his revolutionary paper, he described the three cornerstones of our current minimal invasive techniques: minimal tissue trauma and pain with trocar placement, use of a transparent medium in the cavity and the need for a scope thin enough to be placed through the trocar [2]. It was not until four decades later that a panoramic view of the thorax was achieved through the use of a light source and red lens with micro cameras and video systems. Whilst initially these were in use for diagnostic purposes only, in the mid-1980s this led to the application of these principles to thoracic surgery $[1,2]$. The traditional open approach to lobectomy and other procedures is associated with significant pain that may be long-lasting in duration, primarily from rib-spreading. Video-assisted thoracic surgery (VATS) provided the potential advantage of minimising access and therefore post-operative pain. By the mid 1990s interest in VATS grew and it took over as the preferred technique for many operations including lobectomy [1]. Compared to open thoracotomy, VATS has been shown to minimise post-operative pain, reduce post-operative complications, reduce the duration of chest tube use, limit length of stay, and improve post-operative quality of life of patients [1]. It has become so widely practiced that it is no longer novelty, but rather a conventional approach to performing thoracic surgery [3].

The original practice of three access ports in a 'baseball diamond' pattern in traditional approach to VATS however has been modified over time, to 'next generation' approaches such as 2-port VATS and uniportal procedures [3]. This has led to significant implications as to how the thoracic surgeon ought to master and execute the full potential of the uniportal technique. The use of the da Vinci ${ }^{\circledR}$ robot system (intuitive Surgical, Sunnyvale, CA) in minimally invasive thoracic surgery has gained popularity also due to its ability to enable 3D vision, improve dexterity, enable steady use of instruments, and increase precision in the area of interest. 
However, this precision comes at the expense of at times requiring an extra fourth port [3].

\section{Indications for Lobectomy}

Indications for VATS lobectomy remain controversial. VATS is generally selected in cases where patients are due to undergo surgical management of early stage cancer (TNM stages I and II) with no signs of lymph node invasion [4]. Other indications include: benign disease requiring implementation of anatomical resection, malignant disease with tumour size $<6 \mathrm{~cm}$, localisation in the periphery or $>1 \mathrm{~cm}$ from a fissure or $>3 \mathrm{~cm}$ from the lobar carina. VATS lobectomy may also be performed for solitary metastasis of extrapulmonary cancer which cannot be removed with a typical wedge resection [5]. Pulmonary function values are an important eligibility criterion as single-lung ventilation is a requirement for VATS lobectomy [4]. Clinical judgement should be implemented; however, patients have been reported to have undergone VATS lobectomy with a predicted post-operative FEV1 <30\% [4]. As discussed, selecting suitable patients for VATS lobectomy therefore involves assessing the size and location of the pulmonary lesions, staging the malignant disease, assessing mediastinal lymph node involvement and reviewing the patient through thorough clinical history and examination [5].

Current guidelines formulated by the British Thoracic Society (BTS) and Society of Cardiothoracic Surgeons of Great Britain and Ireland (SCTS) recommend that in terms of patient selection co-morbidity, pulmonary function, cardiovascular fitness and performance status are amongst the criteria requiring consideration pre-operatively prior to selecting patients who may benefit from undergoing a lobectomy [6]. These criteria are summarised in Table 1. Conversion to open thoracotomy from thoracoscopic lobectomy is often indicated in the presence of adhesions, presence of lymph node metastases, large size tumours and bleeding [7].

Table 1: Guidelines for the selection of patients with lung cancer for surgery; adapted for lobectomy - British Thoracic Society of Cardiothoracic Surgeons of Great Britain Ireland Working Party.

1) Age:

a. Perioperative morbidity increases with advancing age. Elderly patients undergoing lung resection are more likely to require intensive perioperative support. Pre-operatively, a careful assessment of co-morbidity needs to be made.

b. Surgery for clinically stage I and II disease can be as effective in patients over 70 years as in younger patients. Such patients should be considered for surgical treatment regardless of age.

c. Age over 80 alone is not a contraindication to lobectomy or wedge resection for clinically stage I disease.

d. Pneumonectomy is associated with a higher mortality risk in the elderly. Age should be a factor in deciding suitability for pneumonectomy.

\section{2) Pulmonary Function}

a. There should be formal liaison in borderline cases between the referring chest physician and the thoracic surgical team.

b. No further respiratory function tests are needed for a lobectomy if the post-bronchodilator FEV1 is $>1.5$ litres and for a pneumonectomy if the postbronchodilator FEV1 is $>2.0$ litres, providing there is no evidence of interstitial lung disease, or unexpected disability due to shortness of breath.

\section{3) High Risk Patients}

a. The management of high-risk patients should be considered at a formal multidisciplinary meeting between a chest physician, surgeon and an oncologist.

b. Such patients should be considered for a a more limited resection; radiotherapy/chemotherapy.

\section{4) Cardiovascular Fitness}

a. All patients for lung resection should have a preoperative ECG.

b. All patients with an audible cardiac murmur should have an echocardiogram.

c. Patients who have had a myocardial infarction within 6 months and is being assessed for thoracic surgery should have a cardiology opinion.

d. Patients who have had coronary bypass surgery should not be precluded from having lung resection. They should be assessed as for other patients with possible risk factors.

e. Patients found to have significant lesions on coronary angiography should be considered for coronary artery bypass surgery before resection.

f. Patients who are at intermediate risk with reasonable functional capacity (able to comfortably walk one flight of stairs) should not normally be regarded as at greater than average risk for postoperative complications. They do not need further cardiac testing.

g. Patients with poor functional capacity and those in whom there is doubt about the degree of severity of their angina pectoris or who report breathlessness which may be due to cardiac disease should have an ECG monitored exercise test and echocardiogram and the results should be discussed with a cardiologist.

h. Patients who are considered to be 'minor risk' should not be considered to be at a greater than average risk for postoperative complications.

i. All patients with a history of previous stroke, transient ischaemic attacks, or carotid bruits should be assessed by carotid Doppler studies. Patients with evidence of significant stenoses (for example over 70\%) should be assessed by a vascular surgeon or consultant in stroke medicine preoperatively and their management discussed with a thoracic surgeon.

\section{5) Weight Loss, Performance Status And Nutrition}

a. Patients presenting with a preoperative weight loss of $10 \%$ or more and/or a performance status of WHO 2 or worse are highly likely to have advanced disease and to require particularly careful staging assessment, together with a search evidence of co-morbidity.

b. Routine preoperative assessment should include a simple measure of nutritional status such as the body mass index, together with a serum albumin measurement. Low values should be accepted as conveying as increased risk of postoperative complications. 


\section{6) Multiple Risk Factors}

a. Patients anatomically suitable for resection but with more than one adverse medical factor should have their management discussed formally at a multidisciplinary meeting between a chest physician, a surgeon and an oncologist.

7) Operability

a. All patients being considered for surgery should have a plain chest radiograph and a computed tomographic (CT) scan of the thorax including the liver and adrenal glands.

b. Confirmatory diagnostic percutaneous needle biopsy in patients presenting with peripheral lesions is not mandatory in patients who are otherwise fit, particularly if there are previous chest radiographs showing no evidence of a lesion.

c. Patients with mediastinal nodes greater than $1 \mathrm{~cm}$ in short axis diameter on the CT scan should undergo biopsy by staging mediastinoscopy, anterior mediastinoscopy or needle biopsy as appropriate.

d. Patients with stage I (cT1N0 and c2T2N0) and stage 2 (cT1N1, cT2N1, cT3N0) tumours should be considered operable).

e. Patients known pre-operatively to have stage IIIA (cT3N1 and cT1-3N2) tumours have a low chance of being cured by surgery alone but might be considered operable in the context of a trial surgery and adjuvant chemotherapy.

f. There is no place for postoperative radiotherapy following complete primary tumour resection.

The indications for elective open lobectomies include surgeon's preference, tumour location (hilar adenopathy) risking complicating the process of vascular dissection or increasing the possibility of vessel injury [8]. Open lobectomies are also performed in cases where VATS is contraindicated such as inability of selective ventilation, extensive growth, previous thoracic surgery, extensive pleural adhesions, previous chemoradiotherapy for lung cancer, and defunct fissures between the lobes amongst the reasons mentioned previously [5].

Robotic technology aims to overcome the restrictions of manual video-thoracoscopy, maintaining the advantages of low invasiveness [9]. The application of robotic assisted thoracic surgery (RATS) however still remains controversial due to surgical factors such as longer operating times, the process of gaining experience and developing the skills required to perform the procedure, cost control, and the lack of data accessible regarding oncological radicality and survival with adequate follow-up period [9]. In regards to indications, most robotic lung resections are performed in patients with TNM staging I and II, with no comorbidities and in lesions that are less than $5 \mathrm{~cm}$. Recently however, some authors have extended the inclusion criteria for RATS and have treated with a robotic approach patients with advanced stages as clinical IIIA stage after neoadjuvant therapies [10], or have performed a sleeve lobectomy or robotic bronchoplastic upper lobectomy [11].

\section{Lobectomy}

Lobectomies can be performed through a posterolateral thoracotomy, muscle sparing thoracotomy, axillary thoracotomy or by VATS. Anatomic lobectomy is the standard of care for pulmonary resection of primary non-small cell lung cancer (NSCLC) [12]. Right-sided lobectomies involve creating a right posterolateral thoracotomy incision if an open-approach is considered [12]. A right upper lobectomy involves dividing the pulmonary vein, followed by the pulmonary artery. After division of the vessels, the right upper lobe is retracted anteriorly, and the right upper lobe bronchus is identified as it takes off from the right main stem. Peri-bronchial lymph nodes are removed, and the bronchus is divided with a stapler or with a knife and closed with Vicryl sutures. The major and minor fissures are completed with a stapler [12]. Right middle lobectomies involve the same procedure of dissecting the vessels, however bronchus dissection and completion of fissures differs whereby a central approach can be undertaken; this is where the middle lobe bronchus is identified after division of the anteriorly located middle lobe pulmonary artery [12]. Alternatively, an anterior approach may be preferable; this is where the middle lobe bronchus is identified first after division of the middle pulmonary vein. Regardless of approach, to finalise the main bronchus is divided with a stapler or cut and closed with absorbable suture. Care is advised to be taken not to impinge the lower lobe bronchus [12]. To finalise the inferior major and minor fissures are completed with a stapler. Right lower lobectomies also follow the same surgical principle. Left-sided lobectomies are more technically demanding than right-sided lobectomies due to the location of the pulmonary artery in relation to the aortic arch and the pulmonary artery branches - particularly the anterior segmental artery. The anterior segmental artery is also known as the 'artery of sorrow' due to it being vulnerable as it is a short but wide branch of the left main pulmonary artery that can be easily avulsed by overvigorous retraction, and if the tear extends proximally, it may force a pneumonectomy [12].

Apart from the catastrophic possible injury to the pulmonary artery during left upper lobectomies through an open approach, conversion to thoracotomy or elective thoracotomy despite anatomical focus brings its own complications [13]. These are outlined in Table 2. The main advantage that VATS has over open thoracotomy relates to the reduced pain following surgery [4]. Other benefits include: reduced chest drain duration, shorter length of stay and quicker recovery period [4]. Furthermore, VATS has been shown to increase overall survival [14]. A study of 752 patients by Sakutuba et al. [14] showed that overall survival rate five-years after surgery was $72 \%$ in the open group vs. $82 \%$ in the VATS group. The disease-free survival was $68 \%$ in the open group vs $80 \%$ in the VATS group. This paper also showed that VATS had overall a shorter median operative time of 117.5 minutes, compared to open which was 171.5 minutes. Also, it has lower chest tube drainage $(987 \mathrm{~mL}$ in VATS vs $1504 \mathrm{~mL}$ in open lobectomy), and shorter length of stay (4.5 
days in VATS, and 7 days in open) [14]. Another study by Whitson et al. [15] supported the above findings by concluding that VATS is an effective, minimally invasive, safe means of resecting lung nodules through lobectomy. This was a retrospective review of 147 consecutive patients undergoing thoracotomy or VATS lobectomy. The study found that VATS patients had significantly less postoperative pneumonia $(\mathrm{p}=0.0023)$. VATS patients were also shown to follow an overall trend of reduced length of hospital stay and fewer chest tube days [15].

Table 2: Possible Complications Post-Thoracotomy.

\begin{tabular}{|c|}
\hline 1) Airway Complications \\
\hline a. Retained secretions \\
\hline b. Aspiration of gastric contents \\
\hline c. Airway oedema/stridor \\
\hline 2) Pulmonary Complications \\
\hline a. Atelectasis \\
\hline b. Bronchospasm \\
\hline c. Tracheobronchial disruption \\
\hline d. Mediastinal shift \\
\hline e. Air leakage \\
\hline f. Lung torsion and infarction \\
\hline g. Bronchopleural fistula \\
\hline h.Pleura effusion, emphysema, chylothorax \\
\hline i.Pneumothorax \\
\hline 3) Other Complications \\
\hline a. Paradoxical motion \\
\hline b. Haematoma \\
\hline c. Wound dehiscence \\
\hline d. Pain \\
\hline e. Mediastinal and subcutaneous emphysema \\
\hline 4) Haemorrhage \\
\hline a. Post-operative bleeding into the pleura \\
\hline b. Bleeding into the tracheobronchial tree \\
\hline 5) Cardiac Complications \\
\hline a. Arrhythmias (mainly atrial fibrillation) \\
\hline b. Pulmonary hypertension, right heart failure \\
\hline c. Cardiac herniation \\
\hline d. Pulmonary thromboembolism \\
\hline e. Myocardial infarction \\
\hline f. Congestive failure \\
\hline 6) Nerve Injuries \\
\hline a. Intercostal nerves \\
\hline b. Phrenic nerve \\
\hline c. Vagus nerves \\
\hline d. Recurrent laryngeal nerve \\
\hline e. Brachial plexus \\
\hline 7) General Complications \\
\hline a. Acute pain \\
\hline b. Respiratory failure \\
\hline Anaesthetic complications. \\
\hline
\end{tabular}

A further study of 202 patients who underwent either VATS lobectomy or thoracotomy lobectomy by Oda et al. [16] further supported the above findings. In this study stage IA NSCLC was confirmed pathologically in 162 patients, of which 60 patients underwent VATS lobectomy, and 102 underwent thoracotomy lobectomy. The study found that the VATS group experienced less blood loss, a shorter duration of chest tube placement, and a shorter postoperative hospital stay [16].

\section{VATS Lobectomy: Multi-Port and Single Port}

Uniportal VATS lobectomy represents the last advancement of minimally invasive techniques in the form of video assisted techniques for the surgical treatment of lung cancer. Uniportal VATS offers the benefit of only compromising one intercostal space whilst enabling direct view to the target tissue [17]. Developments in camera systems, instruments and stapler technology have facilitated this improvement. The parallel instrumentation achieved during the uniportal approach mimics the inside manoeuvres performed during open surgery, and together with the direct view, it facilitates the dissection and division of hilar structures and the fissure. This makes possible the direct transition from open surgery to uniportal VATS [17]. VATS is currently well-established internationally and is performed widely across thoracic centres [3]. The birth of VATS was characterised by its three-port approach without the need to spread the ribs [3]. Overtime the conventional three-port VATS approach was modified to reflect surgical technique in regard to positional factors that initially limited surgeons [3]. In the modified procedure, the camera port was brought more anterior to the anterior axillary line in order to overcome issues of 'mirror imaging' and fencing between camera and the surgeon's instruments commonly noted in the early experience with VATS [3]. The posterior port was also lowered from the anterior to the scapula tip to a lower intercostal level. The utility port position is unchanged [3]. The end result of this modification was a posterior rotation of the initial 'baseball diamond' [3]. Although the diamond shape was preserved, the axis now reflected the umbilicus-towards-shoulder direction and proves to increase procedural comfort. The surgeon now stands on the same side as the camera-holding assistant - thus they both share the same axis, improving surgeon-assistant coordination [3].

After gaining experience with the VATS approach, it was soon realised that the posterior $3 \mathrm{~mm}$ port was not always essential [3]. It was realised that surgical manipulation could be achieved via the utility port alone. The natural progression was therefore to omit that posterior port altogether - leading to the two-port VATS technique. This technique has its limitations in that it requires appropriate surgical technique [3]. Compared to RATS, these newer VATS advancements require no additional equipment as most hospital have incorporated the equipment needed for VATS in their thoracic units, proving these novelty VATS techniques to be advantageous in these aspects. Needlescopic VATS incorporates the use of very fine thoracoscopes $(2-3 \mathrm{~mm}$ in diameter) and instruments $(3-5 \mathrm{~mm}$ diameter) to replace the $10 \mathrm{~mm}$ versions used 
in the conventional VATS equipment [3]. Whilst originally in use for sympathectomy surgery, it has currently gained popularity for lung cancer surgery due to reduced need of torqueing at the wound [3]. Cosmetic appearance is also improved and there is reduced trauma to the intercostal bundles during manipulations [3].

\section{Robotic Assisted Thoracic Surgery (RATS)}

The robotic system consists of a master console used by the surgeon to manipulate the patient cart, connected via electrical cables and optic fibres with three instrumental arms and a camera arm [18]. The surgeon's movements are transmitted to the cart manipulating master handles with a highly sensitive sensor able to filter physiologic hands tremor (6Hz-motion filter) [18]. The surgeon's vision of the area of focus is improved due to the 3D high definition camera. Due to the seven degrees of freedom offered by the robotic instruments, the movements of the human wrist are replicated as they move in the chest cavity [18]. Pitch, yaw and insertion are given by the cart arm; whilst the internal pitch, internal yaw, rotation and grip are guaranteed by the tip of the instrument called in fact Endo Wrist [18]. The daVinci system is currently the only complete surgical system in the market to perform thoracic surgery [18]. Robotic surgery addresses some of the shortcomings of VATS by providing improved dexterity and visualisation. It does this by combining three-dimensional imaging and greater degrees of freedom regarding dexterity [18]. Despite an increase in range of motion, there is a reduction in tactile sensation which is considered to be a disadvantage. RATS also have the disadvantage of a lack of haptic feedback, which may lead to tissue damage, particularly with inexperienced surgeons [18]. Despite this, results for RATS have shown that it is a safe procedure performed by thoracic surgeons with no evident differences in morbidity and mortality $[19,20,21]$. Reviewing 8,253 RATS lobectomies in the Healthcare Cost and Utilisation Project National Inpatient Sample, Tchouta et al. [22] found that high volume centres had a shorter length of stay and decreased mortality.

Other studies have reported significant differences in outcomes compared to VATS. A meta-analysis of 7,438 patients incorporating 14 studies by Liang et al. [23] showed that the 30-day mortality was $0.7 \%$ for RATS, versus $1.1 \%$ for VATS. Furthermore, it found that conversion to thoracotomy was lower in RATS by $1.6 \%$. It was found that there were no significant differences in post-operative complications, time, length of stay or chest tube removal. The only recognised disadvantage with RATS has been the increased operative times, as reported by Louie et al. in an evaluation of 1,220 robotic and 12,378 VATS lobectomies [24]. The benefits regarding RATS and post-operative lack of pain are unfortunately not as direct. In a study of 498 patients who underwent RATS (74), VATS (227) ad open (201) anatomic pulmonary resection, including lobectomy and segmentectomy, Kwon et al. [25] found no significant difference in acute or chronic pain and consequent morphine use between patients who had undergone VATS and RATS lobectomy.
Interestingly, despite no significant difference in pain scores, $69.2 \%$ of patients who received RATS felt the approach affected pain versus $44.2 \%$ VATS $(P=.0330)[25]$. The study suggested that perhaps in patients undergoing RATS, there is a difference between reality and perception allowing them to adjust their approach to pain more positively [25].

\section{VATS Microlobectomy - Is It the Future Of VATS?}

Microlobectomy is one of the latest techniques currently under evaluation [26]. It was designed by a group of VATS lobectomists internationally and it is considered to offer some of the advantages that VATS lobectomies offer [26]. The proposed technique does not differ greatly from the conventional VATS lobectomy. It uses $\mathrm{CO}_{2}$ insufflation, allowing more room to be available in the hemithorax easing lung collapse operatively [26]. This proves advantageous in patients with concomitant air-trapping. Furthermore, it facilitates the dissection and safe placement of a subxiphoid port. It also enables the $\mathrm{CO}_{2}$ to be turned off if desirable by surgeon preference.

Operatively, it involves intubating the patient with single lung isolation and positioning them in the standard lateral position [26]. The main modification is that the xiphisternum, costal margins and the midline down to the umbilicus is marked prior to positioning [26]. Good access to the subxiphoid is then ensured. For patients undergoing an anterior approach lobectomy, the first port is placed in the $4^{\text {th }}$ intercostal space between the inferior angle of the scapula and the nipple. In a conventional VATS this is the area where the utility incision is made. In microlobectomies, a $5 \mathrm{~mm}$ port is inserted here [26]. Chest entry is gained under vision with the Kii-Fios first entry port (Applied Medical, California, USA) with $\mathrm{CO}_{2}$ running at 5L per minute [26].

The camera is placed in the centre of the clear plastic trocar and the port is inserted under vision. If there are adhesions, the $\mathrm{CO}_{2}$ is able to facilitate their separation from the chest wall - an advantage over conventional VATS lobectomy [26]. Once inside the chest, the hemithorax is insufflated at a pressure of 5-10 mmHg. Flow rates of $\mathrm{CO}_{2}$ should be adjusted at this point to avoid hypercarbia, high airway pressures or hypotension [26]. The camera is then directed down to look at the inferior border of the sternum and the anteromedial diaphragm. A $20 \mathrm{~mm}$ skin incision is made vertically below the xiphisternum, then under vision of the soft tissue is dissected down to the tip of the xiphisternum which marks the cranial portion of the linea alba [26]. This is incised vertically for $15 \mathrm{~mm}$. A finger is then placed cranially directly posterior to the xiphisternum and up behind the sternum as far as possible. The finger is then moved laterally into the hemithorax under direct vision [26]. Once the pleura is breached this can be followed with a $12 \mathrm{~mm}$ port. After the subxiphoid port is placed, two further $5 \mathrm{~mm}$ can be placed based on the surgeon's positioning [26].

The operation is then conducted in the usual fashion using $5 \mathrm{~mm}$ instruments [26]. Retraction can be achieved through the subxiphoid 
port, and stapling can take place using a $12 \mathrm{~mm}$ standard stapling device from the subxiphoid port [26]. At the end of the procedure an endo bag is placed from the subxiphoid port and then once the specimen is in the bag, under vision, the linea alba is extended as per required to facilitate the removal of the tumour [26]. Finally, a chest tube is inserted through the subxiphoid port and the wound is closed [26]. The advantages of this method include their feasibility in patients with adhesions, does not differ in surgical approach to VATS, and the operation allows the surgeon's usual view [26]. 72 patients have currently undergone microlobectomy in 6 hospitals in the UK, US and Denmark [26]. The median operating time was found to be 180 minutes (ranging from 94-285). The median blood loss was shown to be $118 \mathrm{~mL}$ (range $5-800 \mathrm{~mL}$ ). There was a $4.1 \%$ conversion rate due to bleeding and $2.8 \%$ conversion rate to VATS rate by extending a port to become a traditional utility incision [26]. The median hospital stay was 3 days, with $22 \%$ of patients going home on the day after the operation. The complications reported included pneumonia (14\%), prolonged air leak (7\%), atrial fibrillation (4\%) and prolonged intubation (4\%) [26]. Further experience in this novel means of performing lobectomies is required to assess its superiority to other endoscopic lobectomy techniques or to a thoracotomy.

\section{Conclusion}

Minimally invasive techniques have radically changed the way that we approach the management of lung cancer. The constant evolution of surgical techniques has enabled us to begin exploring ways of improving patients' experiences perioperatively and overall outcomes. Whilst conventional VATS, at present, have become a mainstay approach of lung cancer surgery; innovations in this technique have allowed for developments to be made in our current surgical practice. Current observational reported data has shown us that VATS and robotic lobectomy are safe, feasible and reproducible means of managing lung cancer. With further study of deviations of this mainstay in techniques we hope that our innovations in surgical techniques also translate in better postoperative outcomes.

\section{References}

1. Alan D L Sihoe (2012) The Evolution of VATS Lobectomy. Topics in Thoracic Surgery Paulo F Guerreiro Cardoso, Intech Open.

2. Loddenkemper R, Mathur PN, Lee P (2011) History and clinical use of thorascopy/pleuroscopy in respiratory medicine. Breathe 8(2): 144155.

3. Sihoe AD (2014) The evolution of minimally invasive thoracic surgery: implications for the practice of uniportal thoracoscopic surgery. Journal of Thoracic Disease 6(6): 604-617.

4. Dziedzic D, Orlowski T (2015) The Role of VATS in Lung Cancer Surgery: Current Status and Prospects for Development. Minimally Inavisve Surgery (2015): 938430

5. Hytych V, Horazdovsky P, Pohnan R, Pracharova S,Taskova A, et al. (2015) VATS lobectomy, history, indication, contraindication and general techniques. Bratislava Medical Journal 116(7): 400-403.

6. (2001) Guidelines on the selection of patients with lung cancer for surgery. Thorax 56: 89-108.
7. Li Y, Wang J, Yang F, Liu J, Li J, et al. (2012) Indications for conversion of thoracoscopic to open thoracotomy in video-assisted thoracoscopic lobectomy. ANZ Journal of Surgery 82(4): 245-250.

8. Amore D, Di Natale D, Scaramuzzi R, Curcio C (2018) Reasons for conversion during VATS lobectomy: what happens with increased experience. The Journal of Visualized Surgery 4(53): 1-5.

9. Ricciardi S, Cardillo G, Zirafa CC, Davini F, Melfi F, et al. (2017) Robotic lobectomies: when and why? Journal of Visualized Surgery 3(112).

10. Schmid T, Augustin F, Kainz G, Pratschke J, Bodner J, et al. (2011) Hybrid video-assisted thoracic surgery-robotic minimally invasive right upper lobe sleeve lobectomy. The Annals of Thoracic Surgery 91(6): 19611965.

11. Nakamura H, Taniguchi Y, Miwa K, Fujioka S, Matsuoka Y, et al. (2013) A successful case of robotic bronchoplastic lobectomy for lung cancer. Annals of Thoracic and Cardiovascular Surgery 19(6): 478-80.

12. Chilkwe J, Cooke D, Weiss A (2013) Oxford Specialist Handbooks in Surgery - Cardiothoracic Surgery, 2nd ed. Oxford: Oxford University Press.

13. Sengupta S (2015) Post-operative pulmonary complications after thoracotomy. Indian Journal of Anaesthesia 59(9): 618-626.

14. Sakuraba M, Miyamoto H, Oh S, Shiomi K, Sonobe S, et al. (2007) Videoassisted thoracoscopic lobectomy vs. conventional lobectomy via open thoracotomy in patients with clinical stage IA non-small cell lung carcinoma. Interactice Cardiovascular and Thoracic Surgery 6(5): 614617.

15. Whitson BA, Andrade RS, Boettcher A, Bardales R, Kratzke RA, et al. (2007) Video-assisted thoracoscopic surgery is more favorable than thoracotomy for resection of clinical stage I non-small cell lung cancer. The Annals of Thoracic Surgery 83(6): 1965-1970.

16. Oda R, Okuda K, Osaga S, Watanabe T, Sakane T, et al. (2018) Longterm outcomes of video-assisted thoracoscopic surgerylobectomy vs. thoracotomy lobectomy for stage IA non-small cell lung cancer. Surgery Today 49(5): 369-377.

17. De La Torre M, González-Rivas D, Fernández R, Delgado M, Fieira E, Méndez L (2016) Uniportal VATS lobectomy. Minerva Chirurgica 71(1): 46-60.

18. Lin J (2017) Robotic lobectomy: revolution or evolution? The Journal of Thoracic Disease 9(9): 2876-2880.

19. Swanson SJ, Miller DL, McKenna RJ Jr, Howington J, Marshall MB, et al. (2014) Comparing robot-assisted thoracic surgical lobectomy with conventional video-assisted thoracic surgical lobectomy and wedge resection: results from a multihospital database (Premier). Journal of Thoracic and Cardiovascular Surgery 147(3): 929-937.

20. McKenna RJ Jr (2008) Complications and learning curves for videoassisted thoracic surgery lobectomy. Thoracic Surgery Clinics 18(3): 275-280.

21. Fahim C, Hanna W, Waddell T, Shargall Y, Yasufuku K, et al. (2017) Robotic-assisted thoracoscopic surgery for lung resection: the first Canadian series. Canadian Journal of Surgery 60(4): 260-265.

22. Tchouta LN, Park HS, Boffa DJ, Blasberg JD, Detterbeck FC, et al. (2017) Hospital Volume and Outcomes of Robot-Assisted Lobectomies. Chest 151(2): 329-339.

23. Liang H, Liang W, Zhao L, Chen D, Zhang J, et al. (2018) Robotic Versus Video-assisted Lobectomy/Segmentectomy for Lung Cancer: A Metaanalysis. Annals of Surgery 268(2): 254-259.

24. Louie BE, Wilson JL, Kim S, Cerfolio RJ, Park BJ, et al. (2016) Comparison of Video-Assisted Thoracoscopic Surgery and Robotic Approaches for Clinical Stage I and Stage II Non-Small Cell Lung Cancer Using The Society of Thoracic Surgeons Database. Annals of Thoracic Surgery 102(3): 917-924. 
25. Kwon ST, Zhao L, Reddy RM, Chang AC, Orringer MB, et al. (2017) Evaluation of acute and chronic pain outcomes after robotic, videoassisted thoracoscopic surgery, or open anatomic pulmonary resection. Journal of Thoracic and Cardiovascular Surgery 154(2): 652-659.

\section{ISSN: 2574-1241}

DOI: 10.26717/BJSTR.2019.19.003244

Amer Harky. Biomed J Sci \& Tech Res

(C) This work is licensed under Creative Commons Attribution 4.0 License

Submission Link: https://biomedres.us/submit-manuscript.php
26. Mydin MIM, El-Saegh MM, Nardini M, Dunning J (2017) VATS, robotic lobectomy and microlobectomy-the future is just ahead? Video-Assisted Thoracic Surgery 2(14): 1-8.

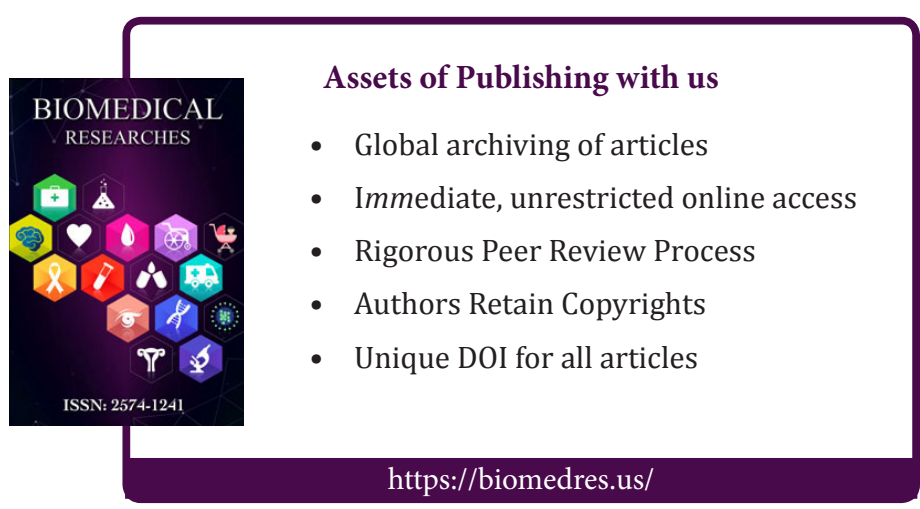

